\title{
On 3-chromatic distance-regular graphs
}

\author{
Aart Blokhuis • Andries E. Brouwer • \\ Willem H. Haemers
}

Received: 30 November 2006 / Accepted: 13 June 2007 / Published online: 28 July 2007

(C) Springer Science+Business Media, LLC 2007

\begin{abstract}
We give some necessary conditions for a graph to be 3-chromatic in terms of the spectrum of the adjacency matrix. For all known distance-regular graphs it is determined whether they are 3-chromatic. A start is made with the classification of 3-chromatic distance-regular graphs, and it is shown that such graphs, if not complete 3-partite, must have $\lambda \leq 1$.
\end{abstract}

Keywords Distance-regular graphs $\cdot$ Chromatic number

\section{AMS Classification $05 \mathrm{E} 30 \cdot 05 \mathrm{C} 15$}

\section{Introduction}

Throughout the paper $\Gamma$ is a graph without loops on $v$ vertices with adjacency matrix $A$ and eigenvalues $\lambda_{1} \geq \cdots \geq \lambda_{v}$. A proper coloring of $\Gamma$ is a partition of the vertex set of $\Gamma$ into cocliques (i.e., independent sets of vertices). Such cocliques are called color classes. The chromatic number $\chi(\Gamma)$ of $\Gamma$ is the minimum number of color classes in a proper coloring.

If $\chi(\Gamma) \leq 2$, then $\Gamma$ is bipartite. Bipartite graphs are easily recognized and there is a characterization in terms of the eigenvalues (see [6]):

A. Blokhuis · A. E. Brouwer

Department of Mathematics, Technological University Eindhoven, Eindhoven, The Netherlands

A. Blokhuis e-mail: aartb@win.tue.nl

A. E. Brouwer

e-mail: aeb@cwi.nl

W. H. Haemers $(\bowtie)$

Department of Econometrics \& O.R., Tilburg University, Tilburg, The Netherlands e-mail: haemers@uvt.nl 
Proposition $1.1 \chi(\Gamma) \leq 2$ if and only if $\lambda_{i}=-\lambda_{v-i+1}$ for $i=1, \ldots, v$.

However, for graphs with a given chromatic number greater than 2, there is no characterization in terms of the spectrum. Indeed, there exist pairs of graphs with the same spectrum, but different chromatic number (for example the complement of the $4 \times 4$ grid and the complement of the Shrikhande graph). Moreover, if $\chi \geq 3$ it is an NP-complete problem to decide whether a given graph has chromatic number $\chi$ (see [13]).

In this paper we will present some necessary eigenvalue conditions for being 3-chromatic. Then we will look at distance-regular graphs, determine for all known ones whether they are 3 -chromatic, and make a start with the classification of all 3-chromatic distance-regular graphs.

The classification of all bipartite distance-regular graphs is hopeless.

We use [3] as a general reference on distance-regular graphs.

\section{Eigenvalue conditions}

\subsection{Hoffman colorings}

For general graphs, Hoffman [16] proved the following lower bound for the chromatic number:

Theorem 2.1 If $\Gamma$ has at least one edge, then $\chi(\Gamma) \geq 1-\lambda_{1} / \lambda_{v}$.

When equality holds we call the coloring a Hoffman coloring. Note that by Proposition 1.1, all 2-chromatic graphs have a Hoffman coloring. For a $k$-regular graph Hoffman (unpublished, see [3]) proved:

Proposition 2.2 If $\Gamma$ is $k$-regular, and $C$ is a coclique in $\Gamma$, then

$$
|C| \leq v \frac{-\lambda_{v}}{k-\lambda_{v}} .
$$

Equality implies that every vertex not in $C$ has exactly $-\lambda_{v}$ neighbors in $C$.

If $C$ is the largest color class in a coloring of $\Gamma$ with $\chi(\Gamma)$ colors, then clearly $\chi(\Gamma)|C| \geq v$. Since $k=\lambda_{1}$, Proposition 2.2 gives the same lower bound for $\chi(\Gamma)$ as Theorem 2.1, but in this case we have more information about the case of equality.

Proposition 2.3 If $\Gamma$ is regular, then all color classes of a Hoffman coloring have equal size, and the color partition is equitable (that is, each vertex $x$ has exactly $-\lambda_{v}$ neighbors in each color class not containing $x$ ). Moreover, the multiplicity of $\lambda_{v}$ is at least $\chi(\Gamma)-1$ and equality implies that $\Gamma$ has a unique coloring with $\chi(\Gamma)$ colors (up to permutation of the colors).

Proof All color classes are cocliques for which Hoffman's coclique bound is tight. So the first sentence follows from Proposition 2.2. A vector that is 1 on one color class, -1 on another, and 0 elsewhere, is an eigenvector with eigenvalue $\lambda_{v}$. This shows that the eigenvalue $\lambda_{v}$ has multiplicity at least $\chi(\Gamma)-1$. If there is another coloring with $\chi(\Gamma)$ colors, we find eigenvectors that are not in the span of those just found.

In fact, the last sentence of the above proposition is also true for a Hoffman coloring in a non-regular connected graph. 


\subsection{3-chromatic graphs}

Hoffman's theorem for the chromatic number implies that if $\Gamma$ has chromatic number 3 then $\lambda_{1} \leq-2 \lambda_{v}$. In [15, p. 22] more bounds of a similar nature were obtained, and especially in case of small chromatic numbers, they can be useful (see Sect. 2.3 for an alternative proof).

Theorem 2.4 If $\Gamma$ has chromatic number 3 , then

$$
2 \lambda_{1+\ell} \geq-\lambda_{v-2 \ell} \text { and } \lambda_{1+2 \ell} \leq-2 \lambda_{v-\ell}(0 \leq \ell \leq v / 3) .
$$

For example, let $\Gamma$ be the folded 11-cube (with $d=5$ and $v=1024$ ). The spectrum of $\Gamma$ (see [3]) is $11^{1}, 7^{55}, 3^{330},-1^{462},-5^{165},-9^{11}$ (with multiplicities written as exponents). So $\lambda_{v-176}=-1$ and $\lambda_{353}=3$, and the second inequality of this theorem is violated for $\ell=176$. So $\chi(\Gamma)>3$. This argument works for folded $(2 d+1)$-cubes when $d \leq 5$, but unfortunately not for $d \geq 6$. See Theorem 3.10 below for a proof that the chromatic number of the folded $(2 d+1)$-cube is 4 for all $d$.

Proposition 2.5 Let $\Gamma$ be 3-chromatic, and let $C$ be any color class in a 3-coloring.

i. If $\Gamma$ is $k$-regular then $|C| \geq v\left(k+\lambda_{v}\right) /\left(2 k+\lambda_{v}\right)$. Equality implies that all vertices outside $C$ have the same number of neighbors in $C$.

ii. $\lambda_{i} \geq-\lambda_{v+1-i-|C|}$ and $\lambda_{v+1-i} \leq-\lambda_{i+|C|}$ for $1 \leq i \leq v-|C|$.

Proof Consider the bipartite subgraph $\Gamma^{\prime}$ of $\Gamma$ induced by the remaining two color classes. Put $c=|C|$ and let $\lambda_{1}^{\prime} \geq \cdots \geq \lambda_{v-c}^{\prime}$ be the eigenvalues of $\Gamma^{\prime}$.

If $\Gamma$ is $k$-regular, $\Gamma^{\prime}$ has $k v / 2-k c$ edges, hence $\lambda_{1}^{\prime} \geq k(v-2 c) /(v-c)$ with equality if and only if $\Gamma^{\prime}$ is regular. Using $\lambda_{v} \leq \lambda_{v-c}^{\prime}=-\lambda_{1}^{\prime}$, statement $i$ follows.

Eigenvalue interlacing gives $\lambda_{i} \geq \lambda_{i}^{\prime}=-\lambda_{v-c+1-i}^{\prime} \geq-\lambda_{v+1-i-c}$ and $\lambda_{v+1-i} \leq$ $\lambda_{v-c+1-i}^{\prime}=-\lambda_{i}^{\prime} \leq-\lambda_{i+c}$.

For example, consider the unique distance-regular graph with intersection array $\{15,14,12 ; 1,1,9\}$. This graph has 506 vertices and spectrum $15^{1}, 4^{230},-3^{253},-8^{22}$. If there is a 3 -coloring, then $|C| \leq 168$ for some color class $C$. But then the second inequality of Proposition 2.5.i $i$ with $i=23$ gives $-3=\lambda_{484} \leq-\lambda_{|C|+23}=-4$. Therefore this graph is not 3-colorable.

Theorem 2.6 Suppose $\Gamma$ is a 3-chromatic edge-regular graph with parameters $(v, k, \lambda)$ (i.e., $\Gamma$ is $k$-regular and every edge is in exactly $\lambda$ triangles). If $\lambda \neq 0$ then every 3 -coloring is a Hoffman coloring.

Proof Let $A$ and $N$ be the adjacency matrix and the vertex-triangle incidence matrix of $\Gamma$, respectively. Then $N N^{\top}=\lambda A+\frac{1}{2} k \lambda I$. Since $N N^{\top}$ is positive semi-definite, $\lambda_{v} \geq-k / 2$. So Hoffman's bound is tight.

\subsection{The inertia bound}

For a graph $\Gamma$, let $\alpha(\Gamma)$ be the independence number of $\Gamma$, that is, the size of the largest coclique. The following proposition is due to Cvetković (see [6]; it is an immediate consequence of eigenvalue interlacing).

Proposition 2.7 Let $\Gamma$ be a graph of order $v$, and let $B$ be a matrix indexed by the vertex set of $\Gamma$ such that if $x \neq y$ then $B_{x y}=0$. Let $n_{+}(B)$ and $n_{-}(B)$ be the number of positive and negative eigenvalues of $B$, respectively. Then $\alpha(\Gamma) \leq v-n_{+}(B)$ and $\alpha(\Gamma) \leq v-n_{-}(B)$. 
For example, if $\Gamma$ is the unique distance-regular graph with intersection array $\{21,20,16,9,2,1 ; 1,2,3,16,20,21\}$ on $v=2048$ vertices (see [3]), then $\Gamma$ has 1387 positive eigenvalues, so $\alpha(\Gamma) \leq 661<v / 3$. Therefore $\Gamma$ is not 3 -colorable (note that the result also follows from Theorem 2.4).

There are less trivial ways to obtain bounds on the chromatic number from bounds on the independence number, cf. Godsil [14]. Indeed, the independence number of the Cartesian product $K_{m} \times \Gamma$ equals the size of the largest $m$-colorable subgraph of $\Gamma$. In particular, $\Gamma$ is $m$-colorable if and only if $K_{m} \times \Gamma$ has independence number $v$.

Let $r$ be a real number, and consider the Cartesian product $K_{m} \times \Gamma$, where the 'vertical' edges (in a copy of $K_{m}$ ) are weighted $r$ and the 'horizontal' ones (in a copy of $\Gamma$ ) get weight 1 . The weighted adjacency matrix $B$ has eigenvalues $\lambda+(m-1) r$ (once) and $\lambda-r(m-1$ times) for each eigenvalue $\lambda$ of $\Gamma$. Now choose $r$ in such a way that $B$ has as few nonpositive or nonnegative eigenvalues as possible, and obtain a bound on the chromatic number of $\Gamma$.

The technique can be used to give an alternative proof of Theorem 2.4.

Proof (of Theorem 2.4). Suppose that $0 \leq \ell<v / 3$ and $\lambda_{1+2 \ell}>-2 \lambda_{v-\ell}$. If $\lambda_{v-\ell}>0$ then $n_{+}(A) \geq v-\ell$ so that $\alpha(\Gamma) \leq \ell<v / 3$ and $\chi(\Gamma)>3$. So, we may assume $\lambda_{v-\ell} \leq 0$. It follows that $\lambda_{1+2 \ell}>0$. Use $m=3$ and $r$ with $-\frac{1}{2} \lambda_{1+2 \ell}<r<\lambda_{v-\ell}$. Then the weighted adjacency matrix $B$ has at most $2 \ell+(v-2 \ell-1)=v-1$ nonpositive eigenvalues, so that $\Gamma$ is not 3-chromatic. The other cases go similarly.

Similarly, Proposition 2.5.i i is the special case $m=2$ of this argument: if there is a 3 -coloring with color class $C$, then the independence number of $K_{2} \times \Gamma$ is at least $v-|C|$.

\section{Distance-regular graphs}

Assume $\Gamma$ is a distance-regular graph with diameter $d$, intersection array

$$
\left\{b_{0}, \ldots, b_{d-1} ; c_{1}, \ldots, c_{d}\right\}
$$

and degree $k=b_{0}$. As usual we define $b_{d}=c_{0}=0, k=b_{0}, a_{i}=k-b_{i}-c_{i}$ for $i=0, \ldots, d, \lambda=a_{1}$, and $\mu=c_{2}$.

\subsection{Diameters 1 and 2}

If $d=1$, the triangle $K_{3}$ is the only 3-chromatic distance-regular graph. A distance-regular graph with $d=2$, is a connected strongly regular graph. The 3-chromatic strongly regular graphs are easily determined (see [15]):

Theorem 3.1 If $\Gamma$ is a connected 3-chromatic strongly regular graph, then $\Gamma$ is the pentagon, the Hamming graph $\mathrm{H}(2,3)$, the Petersen graph $\mathrm{O}_{3}$, or the complete 3-partite graph $\mathrm{K}_{m, m, m}$ $(m>1)$.

In fact, all 4-chromatic strongly regular graphs [15] and almost all 5-chromatic strongly regular graphs [11] have been determined.

\subsection{Generalized polygons}

Theorem 3.2 If $\Gamma$ is the collinearity graph of a generalized $n$-gon $G$ of order $(s, t)$, then $\Gamma$ is 3-chromatic if and only if $s=2$ and $t=1$, or $s=t=1$ and $n$ is odd. 
Proof Assume $\Gamma$ is 3-chromatic. If $s=1$ then $\Gamma$ is an odd cycle. Otherwise it is clear that $s=2$ and $n \geq 4$. If $n=4$, then $\Gamma$ is strongly regular and Theorem 3.1 gives $t=1$. If $n>4$ and $t>1$, then (see [3]) $(n, t)=(6,2),(6,8)$, or $(8,4)$.

If $n=6, t=2$, then $G$ is a generalized hexagon of order 2 (on 63 points). There exist exactly two such hexagons (one is the dual of the other). For both cases De Wispelaere [9] showed that $\Gamma$ is not 3-colorable.

If $n=6, t=8$, then $G$ is the unique generalized hexagon of order $(2,8)$ (on 819 points). This generalized hexagon contains a subhexagon of order 2 , and therefore $\Gamma$ is not 3 -colorable (see [9]).

If $n=8, t=4, G$ is a generalized octagon of order $(2,4)$ (on 1755 points). Then the eigenvalues of $\Gamma$ are

$$
10^{1}, 5^{351}, 1^{650},-3^{675},-5^{78} \text {. }
$$

It follows that $\Gamma$ is not 3 -colorable by Theorem 2.4 (take $\ell=352$ ).

Finally, if $\Gamma$ is the collinearity graph of a generalized $2 m$-gon of order $(2,1)$, then $\Gamma$ is 3-chromatic. Indeed, such a graph is the flag graph of a generalized $m$-gon of order 2 , which is the line graph of the bipartite incidence graph of a generalized $m$-gon of order 2 . These bipartite graphs have valency 3 and hence have edge-chromatic number 3 (by König's theorem), so that their line graphs have chromatic number 3.

\subsection{Near polygons}

A 3-chromatic distance-regular graph with $\lambda>0$ must be similar to a regular near polygon with lines of size 3 .

Theorem 3.3 Let $\Gamma$ be a 3-chromatic distance-regular graph with $\lambda>0$, not a complete tripartite graph $K_{m, m, m}$. Then $\lambda=1$ and $\mu \leq 2$ and $\Gamma$ has no induced 5-cycle and no 2-colored 4-cycle.

Proof Let $W$ (hite), $B(l u e)$ and $R(e d)$ denote the sets of points in the different color classes for a proper 3-coloring of $\Gamma$. By Theorem 2.6 and Proposition 2.3, W, B and $R$ have the same size, and every point in one class has $k / 2$ neighbors in each of the other classes.

Writing down the diagram from a white point $w \in W$, we see that the points in $\Gamma_{1}(w)$ are partitioned into $k / 2$ red and $k / 2$ blue points. For $\Gamma_{2}(w)$ we find a partition into $(k / 2)(k / 2-$ $\lambda) / \mu$ red points, the same number of blue points and $k(k / 2-1) / \mu$ white points.

If $\lambda=k / 2$ then we obtain a complete tripartite graph. If $\lambda<k / 2$, there exists a red-blue edge $\{x, y\}$ between $\Gamma_{1}(w)$ and $\Gamma_{2}(w)$, and any common neighbor of $x$ and $y$ is white and hence in $\Gamma_{2}(w)$. Therefore $a_{2}>0$. If $\lambda>1$ this is impossible since then there are more white vertices in $\Gamma_{2}(w)$ than blue and red together. Therefore $\lambda=1$.

The number of white vertices in $\Gamma_{2}(w)$ equals the number of blue and red together so we get a bipartite graph, and in particular there are no edges between red and blue in $\Gamma_{2}(w)$. It follows that we cannot have 2-colored quadrangles in the graph, for the unique point of the third color adjacent to an edge has to have a further neighbor, but $\lambda=1$. As a consequence $\mu=1$ or 2 . It also follows that we cannot have any induced 5-cycle in the graph, for one of the colors is present only once and opposite to it there is a forbidden edge.

We would like to show that in case $\lambda=1$ we have a regular near polygon or an antipodal cover of one. Some steps in this direction: 
Lemma 3.4 Let $\Gamma$ be a 3-chromatic distance-regular graph with $\lambda=1$, and call its triangles lines. If a point $x$ has distance at most 2 to a line $L$, then there is a unique point on $L$ closest to $x$. In particular, $a_{2}=c_{2}$.

Proof Let us continue with the case $\lambda=1$ in the above proof and consider an adjacent white-blue pair of vertices $p, q$ in $\Gamma_{2}(w)$ and suppose the common red neighbor $r$ is in $\Gamma_{3}(w)$. The blue point $q$ has a red common neighbor $x$ with $w$, and the white point $p$ must have a common neighbor $y$ with $w$, and $x$ and $y$ must be adjacent, otherwise we have an induced pentagon. In particular, $y$ is unique and $\mu=1$. On the other hand, $y, q$ have the two common neighbors $x, p$, contradicting $\mu=1$. So, $r$ is not in $\Gamma_{3}(w)$.

For example, the graph with intersection array $\{6,4,2,1 ; 1,1,4,6\}$, the triple cover of $G Q(2,2)$, is not 3-chromatic.

Lemma 3.5 Let $\Gamma$ be a 3-chromatic distance-regular graph with $\lambda=1$, and call its triangles lines. There are nonzero numbers $u_{i}$ satisfying $u_{0}=1, u_{d+1}=0$ and $c_{i} u_{i-1}+\left(a_{i}+\frac{1}{2} k\right) u_{i}+$ $b_{i} u_{i+1}=0(1 \leq i \leq d)$ such that if $w$ is a point, and $L=\{x, y, z\}$ is a line, and $w$ has distances $h, i, j$ to $x, y, z$, respectively, then $u_{h}+u_{i}+u_{j}=0$. In particular, $x$ does not have the same distance to the three points on $L$.

Proof The smallest eigenvalue of $\Gamma$ is $\theta=-k / 2$. Apply [3, Proposition 4.4.1], to find numbers $u_{i}(0 \leq i \leq d)$ and a representation of the vertices of $\Gamma$ as unit vectors in a Euclidean space such that two vertices at distance $i$ have representing unit vectors with inner product $u_{i}$. Since $\theta$ is the smallest eigenvalue, the $u_{i}$ alternate in sign, and in particular are nonzero. We have $u_{0}=1$ and $u_{1}=-\frac{1}{2}$.

Looking at the Gram matrix of the image of a line $\{x, y, z\}$ we see that the sum of the images of $x, y, z$ is 0 . It follows that if $w$ is any point, and the distances of $w$ to $x, y, z$ are $h, i, j$, then $u_{h}+u_{i}+u_{j}=0$.

Theorem 3.6 A 3-chromatic distance-regular graph with $d=3$ and $\lambda \neq 0$ is one of the following.

i. the flag graph of the Fano plane $(v=21)$,

ii. the Hamming graph $H(3,3)(v=27)$,

iii. the coset graph of the extended ternary Golay code $(v=729)$.

Proof By Lemmas 3.4 and 3.5, $\Gamma$ is (the collinearity graph of) a regular near hexagon, and we saw that $\mu \leq 2$. Now near hexagons with $\lambda=1$ and $\mu \geq 2$ were classified in [4], and only $H(3,3)$ and the near hexagon on 729 points have $\mu=2$. On the other hand, if $\mu=1$, then we have (the collinearity graph of) a generalized hexagon and Theorem 3.2 applies.

It is not true that every 3 -chromatic distance-regular graph with $\lambda=1$ is a near polygon. However, we have the following.

Proposition 3.7 Let $\Gamma$ be a 3-chromatic distance-regular graph with $\lambda=1, \mu=2, d \geq 3$. Then there is a number $e, 3 \leq e \leq d$, such that $a_{i}=c_{i}$ if $i<e, a_{i}=k / 2$ if $i=e$, and $a_{i}=b_{i}$ if $i>e$. If $a_{d}>0$ (equivalently, if $e=d$ ), then $\Gamma$ is a near polygon.

Proof Assume $\lambda=1$ and $\mu=2$. View the graph as the collinearity graph of a geometry with lines of size 3 . Any two vertices at distance 2 determine a quad $3 \times 3$ (that is, $H(2,3)$ ): since $\mu=2$ they determine a quadrangle, and using the fact shown above that if a point 
has distance 2 to two vertices on a line, it has distance 1 to the third point, we see that the quadrangle completes to a quad.

Now apply Lemma 3.5 to find nonzero $u_{i}$ such that if $w$ is any point, and $\{x, y, z\}$ any line, and the distances of $w$ to $x, y, z$ are $h, i, j$, then $u_{h}+u_{i}+u_{j}=0$.

As already observed, the case $h=i=j$ does not occur. This means that two points of the line have the same distance, say $i$, to $w$, and the third point has distance $i+1$ or $i-1$ to $w$. In the former case $2 u_{i}+u_{i+1}=0$, in the latter $2 u_{i}+u_{i-1}=0$.

For small distances we know that only the triples $0,1,1$ and 1,2,2 and 2,3,3 occur, so that $u_{0}=1, u_{1}=-\frac{1}{2}, u_{2}=\frac{1}{4}, u_{3}=-\frac{1}{8}$.

It is impossible to have both $i-1, i-1, i$ and $i, i+1, i+1$, since otherwise $u_{i}=$ $-2 u_{i-1}=-2 u_{i+1}$ and no line $i, i, *$ is possible. Now make a quad on two intersecting lines with patterns $i-1, i-1, i$ and $i, i+1, i+1$, and see that $i, i, i \pm 1$ does occur, contradiction.

So, there is a unique distance $e$, the largest $i \leq d$ such that $u_{i}=\left(-\frac{1}{2}\right)^{i}$, such that if $i<e$ lines with $i, i, *$ have $i, i, i-1$, and if $i>e$ they have $i, i, i+1$.

The graph $\Gamma$ is a near polygon precisely when $u_{d}=-u_{d-1} / 2=\left(-\frac{1}{2}\right)^{d}$. In this case $a_{d}=c_{d}=k / 2$. If it is not a near polygon, then $u_{d}=-2 u_{d-1}$. In this case there are no lines with distance pattern $d, d, *$, and it follows that $a_{d}=0$.

The coset graph of the shortened extended ternary Golay code (that is, the dual of the perfect ternary Golay code) on 729 vertices (see [3, p. 365]) has $\lambda=1, \mu=2, d=5$ and $a_{d}=0$ (hence is not a near polygon), and is 3-chromatic.

Proposition 3.8 Let $\Gamma$ be a 3-chromatic distance-regular graph with $\lambda=1, \mu=2, d>3$, $a_{d}>0$. Then $\Gamma$ is $H(d, 3)$.

Proof (Use notation as in [2]. Here $t_{i}+1=c_{i}$ and $s=2$.) A regular near polygon with $\lambda>0$ and $\mu>1$ contains geodetically closed sub near polygons of all diameters [4]. If the near polygon is 3-chromatic, then by Theorem 3.6 the sub near hexagons ('hexes') must be either $H(3,3)$ or the near hexagon on 729 vertices. According to [7, p. 56], no regular near octagon has hexes isomorphic to the 729-point near hexagon. Indeed, if such a near octagon has $t+1$ lines/point, then by [2] we have $t+1 \leq\left(s^{2}+1\right)\left(t_{3}+1\right)=60$ and for these $t$ the multiplicity of the eigenvalue $-t-1$ is integral only when $t+1=40$. But then the number of hexes on a quad is $\left(t-t_{2}\right) /\left(t_{3}-t_{2}\right)=38 / 10$, contradiction. It follows by induction on $d$ that if $d \neq 3$ we necessarily have $H(d, 3)$. Indeed, let $d \geq 4$. By induction $c_{i}=i$ for $i<d$. We have a quotient of $H(d, 3)$, cf. [3, p. 155]: there is a map from $H(d, 3)$ to $\Gamma$ that is an isomorphism on subgraphs $H(d-1,3)$. The fibers of the quotient map are codes of covering radius $d$ and minimal distance at least $2 d$. A truncated code has covering radius $d-1$ (a point at distance $d$ has a neighbour at distance $d-1$ on each line through it) and minimal distance at least $2 d-1$, so is perfect, and for $d \geq 3$ no such codes exist other than the perfect ternary Golay code.

It should be possible to completely classify the case $\lambda=1, \mu=2$, but we have not done so. Some partial results are: $e>d / 2$, and for $e \geq 4$ there are subgraphs $H(3,3)$, and using those, one finds that $\Gamma$ is a quotient of $H\left(\frac{1}{2} k, 3\right)$. In particular, if $e \geq 4$ then $v$ is a power of 3 .

If $d \geq 4$ and $\lambda=\mu=1$ there is only one known regular near polygon that is not a generalized polygon: the Cohen-Tits near octagon on 315 points. However, this near octagon contains generalized hexagons of order 2 (see [8, p. 461]) and hence its collinearity graph is not 3-chromatic. 
3.4 Known 3-chromatic distance-regular graphs

Below we list the known 3-chromatic distance-regular graphs. We give diameter $d$, number of vertices $v$, the intersection array, and a very short description.

\begin{tabular}{lllll}
\hline & $d$ & $v$ & Intersection array & Comment \\
\hline$a$ & $d$ & $2 d+1$ & $\{2,1, \ldots, 1 ; 1, \ldots, 1\}$ & $(2 d+1)$-cycle \\
$b$ & $d$ & $3^{d}$ & $\{2 d, 2 d-2, \ldots, 2 ; 1,2, \ldots, d\}$ & Hamming graph $3^{d}$ \\
$c$ & $d$ & $\left(\begin{array}{c}2 d+1 \\
d\end{array}\right)$ & $\begin{array}{l}\{d+1, d, d, \ldots, m+1 ; 1,1,2, \ldots, m\} \\
\text { where } m=\lfloor d / 2\rfloor\end{array}$ & Odd graph $O_{d+1}$ \\
& & & $\{2 m, m-1 ; 1,2 m\}(m>1)$ & \\
$d$ & 2 & $3 m$ & $\{4,2,2 ; 1,1,2\}$ & $K_{m, m, m}$ \\
$e$ & 3 & 21 & $\{6,5,2 ; 1,1,3\}$ & flags of PG(2,2) \\
$f$ & 3 & 57 & $\{24,22,20 ; 1,2,12\}$ & Perkel graph \\
$g$ & 3 & 729 & $\{3,2,2,1 ; 1,1,1,2\}$ & Cosets of extd. Golay \\
$h$ & 4 & 28 & $\{4,2,2,2 ; 1,1,1,2\}$ & Coxeter graph \\
$i$ & 4 & 45 & $\{3,2,1,1,1 ; 1,1,1,2,3\}$ & flags of GQ(2,2) \\
$j$ & 5 & 20 & $\{4,2,2,18,2,1 ; 1,2,9,2,2,1,1,1,1,1,2\}$ & cosects of dual Golay \\
$k$ & 5 & 729 & $\{3,2,2,2,1,1,1 ; 1,1,1,1,1,1,3\}$ & flags of GH(2,2) \\
$l$ & 6 & 189 & & Biggs-Smith graph \\
$m$ & 7 & 102 & &
\end{tabular}

\subsubsection{Discussion}

None of these graphs is bipartite, so in order to prove that they are 3-chromatic it suffices to indicate a 3 -coloring. For $d=1$, each of the graphs under $(a),(b),(c)$ is a triangle, and also $K_{1,1,1}$ is a triangle.

(a) For $d \geq 1$ the $(2 d+1)$-cycle is 3 -chromatic.

(b) The Hamming graph $H(d, 3)$ consists of all ternary vectors of length $d$, adjacent when they differ in one coordinate position. One finds a 3-coloring (where the colors are the elements of $\mathrm{GF}(3))$ by assigning to the vector $u$ the color $(u, \mathbf{1})$, where $\mathbf{1}$ is the all- 1 vector.

(c), (h) The Odd graph $O_{d+1}$ consists of all subsets of size $d$ of a $2 d+1$ set $\Omega$, adjacent when they are disjoint. Let $\Omega=\{1,2, \ldots, 2 d+1\}$, then a 3 -coloring is given by coloring all $d$-sets containing 1 red, all remaining $d$-sets containing 2 white, and the remaining $d$-sets blue. The graph $O_{d+1}$ is a Kneser graph, and more generally the chromatic number of Kneser graphs is known. The Coxeter graph is an induced subgraph of $\mathrm{O}_{4}$.

(d) The complete 3-partite graphs $K_{m, m, m}$ has an obvious 3-coloring. The restriction $m>1$ in the table only serves to ensure $d=2$.

$(e),(i),(l)$ A generalized $2 n$-gon of order $(1,2)$ is the flag graph of a generalized $n$-gon of order 2, that is, the line graph of the bipartite point-line incidence graph. The known generalized $n$-gons of order 2 are: if $n=2$ then $K_{3,3}$, giving rise to $H(2,3)$; if $n=3$ then the Fano plane, giving rise to case $e$ ); if $n=4$ then the $\operatorname{Sp}(4,2)$ generalized quadrangle, giving rise to case $i)$; and if $n=6$ then the $G_{2}(2)$ generalized hexagon and its dual, giving rise to case $l$ ). (Note that a hexagon and its dual have the same point-line incidence graph, and hence the same flag graph.) As mentioned before, the incidence graphs are bipartite and regular of degree 3 and therefore have chromatic index 3 (by König's theorem), so that their line graphs have chromatic number 3 . 
(f) The Perkel graph is the unique distance-regular graph with intersection array $\{6,5,2 ; 1,1,3\}$. See [3, p. 402] for the structure (and 3-coloring), and [5] for uniqueness. A slightly nicer description: take vertex set $\mathbf{Z}_{3} \times \mathbf{Z}_{19}$ and join $(i, j)$ to $(i+1, k)$ when $(k-j)^{3}=2^{6 i}$.

$(g),(k)$ If $C$ is a ternary code contained in $\mathbf{1}^{\perp}$, then the coset graph of $C$, where two cosets are adjacent when they have representatives that differ in a single coordinate position, has the 3 -coloring assigning $u+C$ to $(u, \mathbf{1})$. Case $b)$ above is the special case $C=\{0\}$. Case $g$ ) is the case where $C$ is the extended ternary Golay code (a self-dual $[12,6,6]_{3}$ code). The resulting graph is a near hexagon, uniquely determined by its intersection array (see [1]). Case $k$ ) is the case where $C$ is the dual of the perfect ternary Golay code (an $[11,5,6]_{3}$ code). We show below in Theorem 3.9 that also this graph is uniquely determined by its parameters.

$(h),(j),(m)$ The non-bipartite distance-regular graphs of degree 3, except $K_{4}$, are 3 -chromatic by Brooks' theorem. There are exactly four such graphs: the Petersen graph $\mathrm{O}_{3}$, the dodecahedron $(j)$, the Coxeter graph $(h)$ and the Biggs-Smith graph $(m)$.

\subsubsection{Uniqueness}

For each of the above distance-regular graphs uniqueness given the parameters is known now. In [3, p. 365], it is asked whether the graph of case $(k)$ is uniquely determined by its parameters. But it is easy to see that it is.

Theorem 3.9 There is a unique distance-regular graph with intersection array $\{22,20,18,2,1 ; 1,2,9,20,22\}$, namely the coset graph of the dual of the ternary Golay code.

Proof Given a graph $\Gamma$ with this intersection array, one checks that $\Delta:=\Gamma_{1} \cup \Gamma_{5}$, the distance 1-or-5 graph of $\Gamma$, has intersection array $\{24,22,20 ; 1,2,12\}$ and hence (see [1], or [3, Theorem 11.3.1]) is the coset graph of the extended ternary Golay code, the near hexagon on 729 points. Calling the triangles in this graph lines, we have lines that were triangles in $\Gamma$ and lines that were antipodal classes in $\Gamma$. If $C$ and $D$ are antipodal classes, and some point of $C$ is adjacent to some point of $D$, then each point of $C$ is adjacent to some point of $D$, so that $C$ and $D$ are parallel in the near hexagon. Now $\Gamma$ is obtained from $\Delta$ by removing a parallel class of lines, and by transitivity of the group a different choice of parallel class gives an isomorphic graph.

\subsection{Ternary codes}

We saw that the Hamming graph $3^{n}$ is 3 -chromatic. More precisely, given any 3 -coloring of a vertex and its $2 n$ neighbors, there is a unique way to complete that to a 3 -coloring of $3^{n}$. In other words, if the three colors are called $0,1,2$, then any 3 -coloring in which the origin is colored 0 is represented by a \pm 1 -vector $c$, where the color of a vertex $x$ (represented by a ternary vector of length $n)$ is the inner product $(x, c)$.

It follows that the coset graph of a ternary linear code $C$ with minimum distance more than 1 will be 3 -chromatic precisely when $C^{\top}$ contains a \pm 1 -vector, that is, a vector of weight $n$. (Indeed, this coset graph is a quotient of $3^{n}$ where vectors that differ by an element of $C$ are identified. The colorings of the coset graph correspond to the colorings of $3^{n}$ that are constant 
on cosets of $C$.) For example, the coset graph (of diameter 3 ) of the extended ternary Golay code is 3-chromatic, but the (strongly regular) coset graph of the perfect ternary Golay code is not. And the coset graph (of diameter 5) of the dual of the perfect ternary Golay code is again 3-chromatic.

\subsection{Cube-like graphs}

A cube-like graph is by definition a Cayley graph for the elementary abelian group $2^{m}$. According to Payan [18] a cube-like graph does not have chromatic number 3. Let us briefly sketch the argument. First we need the chromatic number of the folded $(2 d+1)$-cube, determined in [19]. The argument used in the second proof is from [10].

Theorem 3.10 The chromatic number of the folded $(2 d+1)$-cube is 4 .

First proof It suffices to show that given a 3-coloring of the folded $(2 d+1)$-cube, we can find a 3-coloring of the folded $(2 d-1)$-cube, since then going down to the folded 3-cube, which is $K_{4}$, yields a contradiction. So, suppose we have a 3 -coloring (with colors $0,1,2$ ) of the $(2 d+1)$-cube, such that antipodes have the same color. In order to color the point $u$ (viewed as binary word of length $2 d-1)$ of the $(2 d-1)$-cube, look at the colors of the two vertices $01 u$ and $10 u$ of the $(2 d+1)$-cube. Color $u$ with $i$ when both have color $i$, or when one has color $i$ and the other $i+1(\bmod 3)$. Then $u$ and its antipode $\bar{u}$ get the same color. And adjacent vertices get different colors: if $u$ and $v$ are adjacent and get the same color $i$, then $01 u, 10 u$ and $01 v, 10 v$ both have colors $i, i+1$ so that $11 u$ and $11 v$ both have color $i+2(\bmod 3)$, contradiction.

Second proof Consider the folded $(2 d+1)$-cube $\Gamma$ defined as the collection of subsets of size at most $d$ of the cyclic group $\mathbf{Z}_{2 d+1}$, adjacent when they differ by a single element, or when they are disjoint $d$-sets. We indicate a vertex-critical subgraph. Put $c_{m, i}=$ $\{i, i+2, \ldots, i+2(m-1)\}$ and $C_{m}=\left\{c_{m, i} \mid i \in \mathbf{Z}_{2 d+1}\right\}$ and $C=\bigcup_{0 \leq m \leq d} C_{m}$, so that $C$ is a set of $1+d(2 d+1)$ vertices $c_{m, i}$ in $\Gamma$. Note that $c_{m, i+1} \sim c_{m+1, i \pm 1}$ and $c_{d, i} \sim c_{d, i \pm 1}$. The edges meeting both $C_{m}$ and $C_{m+1}$ form a $(4 d+2)$-cycle $(1 \leq m \leq d-1), C_{0}$ contains a single point, adjacent to all vertices in $C_{1}$, and $C_{d}$ induces a $(2 d+1)$-cycle.

Now suppose we have a 3-coloring of $C$. In any 3-coloring of a cycle, the number of vertices such that both neighbors have the same color is even. That means that the parity of the number of $i$ such that $c_{m, i-1}$ and $c_{m, i+1}$ have the same color does not depend on $m$ $(1 \leq m \leq d)$. For $m=d$ this parity is even. For $m=1$ it is odd. Contradiction.

It is easy to see that the folded $(2 d+1)$-cube has a 4-coloring.

Theorem 3.11 The chromatic number of a cube-like graph is not 3.

Proof Let $\Gamma$ be a cube-like graph, defined by the difference set $S$ in the abelian group $G=2^{m}$. If the graph is bipartite, its chromatic number is 2 . Otherwise, let $C$ be an odd cycle in $\Gamma$, say $C=\left\{c_{0}, \ldots, c_{2 d}\right\}$. Then $e_{i}=c_{i}+c_{i+1} \in S$ for all $i$ (with indices mod $2 d+1$ ) and $\sum e_{i}=0$. Consider the folded $(2 d+1)$-cube, and map it into $\Gamma$ via $u \mapsto \sum_{i \in u} e_{i}$. This map is a homomorphism: it sends edges to edges. That means that the chromatic number of the folded $(2 d+1)$-cube, which is 4 , is not larger than the chromatic number of $\Gamma$.

If $C$ is a binary code, then the coset graph is cube-like. Consequently, no coset graph of a binary code is 3 -chromatic. 


\subsection{Petersen graphs}

The Petersen graph is the set of 10 pairs of symbols from a 5-set, adjacent when the pairs are disjoint. It has independence number 4 and chromatic number 3 . The five independent sets of size 4 are the sets of four pairs on a given symbol. The twenty 3 -colorings are found by taking two independent sets of size four (they have one vertex $x$ in common) and the remaining triple (the neighbors of $x$ ).

Knowledge of the 3-colorings of the Petersen graph helps in the study of graphs that contain Petersen graphs as subgraph.

Theorem 3.12 Let $\Gamma$ be a triangle-free $k$-regular graph such that any two vertices at distance 2 lie in a unique induced Petersen subgraph. If $\Gamma$ is 3-colorable, then $\Gamma$ is the Petersen graph.

Proof Each vertex lies in $\frac{1}{6} k(k-1)$ Petersen subgraphs, so there are $\frac{1}{60} k(k-1) v$ Petersen subgraphs in $\Gamma$. Fix a 3-coloring of $\Gamma$. It induces a 3-coloring on each Petersen subgraph $P$, and selects from each $P$ the unique point $x_{P}$ of which the three neighbors in $P$ have the same color. A vertex is on average special point in $\frac{1}{60} k(k-1)$ Petersen subgraphs. Pick a vertex $w$ that is special point in at most this number of Petersen subgraphs. The Petersen graphs on $w$ induce a Steiner triple system on the neighbors of $w$. The $k$ points of this Steiner triple system have a 2-coloring with at most $\left\lfloor\frac{1}{60} k(k-1)\right\rfloor$ monochromatic triples. But if $k=2 m+1$ then there are at most $m(m+1)$ non-monochromatic pairs, and each non-monochromatic triple uses two, so there are at most $\frac{1}{2} m(m+1)$ non-monochromatic triples. The total number of triples is $\frac{1}{6} k(k-1)$ and we find $\frac{1}{6} k(k-1) \leq\left\lfloor\frac{1}{60} k(k-1)\right\rfloor+\frac{1}{2} m(m+1)$. It follows that $m=1, k=3$ and $\Gamma$ is the Petersen graph.

Consequently, the unique distance-regular graphs with arrays $\{15,14,12 ; 1,1,9\}(v=506)$, $\{9,8,6,3 ; 1,1,3,8\}(v=280),\{7,6,4,4 ; 1,1,1,6\}(v=330)$ and $\{7,6,4,4,4,1,1,1$; $1,1,1,2,4,4,6,7\}(v=990)$, are not 3-chromatic.

\subsection{Pentagons}

In the previous section we used that every 3-coloring of a Petersen graph has a special vertex. The same is true for every 3 -coloring of a pentagon.

Proposition 3.13 Let $\Gamma$ be a distance-regular graph of diameter at least 2 with $\lambda=0$, $a_{2} \neq 0$. If $\Gamma$ possesses a 3-coloring such that each vertex has equally many neighbors of each of the two other colors, then $k=6$.

Proof Consider a white vertex $w$. It has $k / 2$ red and $k / 2$ blue neighbors. At distance 2 from $w$ there are $\left(\frac{1}{2} k-1\right) k / \mu$ white vertices and $\frac{1}{4} k^{2} / \mu$ blue, and as many red vertices. Inside $\Gamma_{2}(w)$ there are $k(k-1) a_{2} /(2 \mu)$ edges, $k(k-2) a_{2} /(2 \mu)$ with a white vertex, and $k a_{2} /(2 \mu)$ blue-red edges. This means that there are $k a_{2} \mu / 2$ pentagons on $w$ for which $w$ is the only white vertex. Since every pentagon has a unique vertex with a color that occurs only once, the total number of pentagons is $v k a_{2} \mu / 2$. On the other hand, direct counting yields that there are $v k(k-1) a_{2} \mu / 10$ pentagons, so that $k=6$.

An example of this situation is the Perkel graph, which has $d=3, v=57, k=6, \lambda=0$ and is 3-chromatic.

As an application of this proposition we see that a putative distance-regular graph with intersection array $\{8,7,5 ; 1,1,4\}$ cannot be 3 -chromatic. Indeed, such a graph has smallest eigenvalue -4 , and by Proposition 2.2 in any 3 -coloring each vertex has four neighbors of each of the other two colors. 


\subsection{Remaining cases}

In Sect. 3.4 we gave the known 3-chromatic distance-regular graphs. Examining the list of known distance-regular graphs that survive the Hoffman bound $\left(\lambda_{v} \leq-\frac{1}{2} k\right.$ with equality if $\lambda>0$ ) and Theorem 3.3 ( $\lambda \leq 1$ and $\mu \leq 2$ if $\lambda=1$ ), and noting the above discussions about generalized polygons, near polygons, coset graphs of ternary codes, and coset graphs of binary codes (never 3-chromatic), a short list of possible candidates is left.

Four candidates were ruled out in the section on Petersen graphs.

Ad hoc inspection of the Wells graph with $d=4, v=32$ shows that its independence number is 10 , so that it cannot be 3-chromatic.

Ad hoc inspection of the Sylvester graph with $d=3, v=36$ shows that its independence number is 12 , and the union of two disjoint 12-cocliques is regular of valency 3 ; the subgraph on the remaining 12 vertices is regular of valency 1 , so that there is no 3 -coloring.

A 3-coloring of the second subconstituent $\Delta$ (with $d=3, v=42$ ) of the HoffmanSingleton graph $\Gamma$ would be a partition into three 14-cocliques, and require three 15 -cocliques pairwise meeting in 1 point in $\Gamma$. But 15 -cocliques in $\Gamma$ meet in $0,3,5,8$ or 15 points, so $\Delta$ is not 3 -chromatic. (It follows that no antipodal graph of diameter 3 is 3 -chromatic.)

That exhausts all graphs known to us. The tables of [3] also list feasible parameter sets for certain small graphs where no example is known. Only two parameter sets required further inspection. (Nonexistence of graphs with intersection arrays $\{15,14,12,6,1,1 ; 1,1,3,12,14,15\}(d=6, v=1,518)$, and $\{5,4,3 ; 1,1,2\}(d=3$, $v=56$ ) was proved by Ivanov \& Shpectorov [17] and Fon-Der-Flaass [12], respectively.)

A graph with intersection array $\{7,6,6 ; 1,1,2\}(d=3, v=176)$ is not 3-chromatic by Theorem 2.4. And a graph with intersection array $\{8,7,5 ; 1,1,4\}(d=3, v=135)$ was shown to be not 3-chromatic in the section on pentagons.

Acknowledgments We thank Bart De Bruyn and Hennie Wilbrink for information about near polygons and discussions on 3-chromatic distance-regular graphs.

\section{References}

1. Brouwer AE (1982) The uniqueness of the near hexagon on 729 points. Combinatorica 2:333-340

2. Brouwer AE, Wilbrink HA (1983) The structure of near polygons with quads. Geom Dedicata 14:145-176

3. Brouwer AE, Cohen AM, Neumaier A (1989) Distance-regular graphs. Springer-Verlag, Berlin, Heidelbeg, Newyork

4. Brouwer AE, Cohen AM, Hall JI, Wilbrink HA (1994) Near polygons and Fischer spaces. Geom Dedicata 49:349-368

5. Coolsaet K, Degraer J (2005) A computer-assisted proof of the uniqueness of the Perkel graph. Des Codes Cryptogr 34:155-171

6. Cvetković DM, Doob M, Sachs H (1980) Spectra of graphs: theory and applications. Deutscher Verlag der Wissenschaften, Berlin; Academic Press, New York (Third edition, Johann Abrosius Barth Verlag, Heidelberg-Leipzig, 1995)

7. De Bruyn B (2006) Near polygons. Birkhäuser Verlag, Basel Boston Berlin

8. De Clerck F, Van Maldeghem H (1995) Some classes of rank 2 geometries: handbook of incidence geometry. Elsevier, Amsterdam, pp 433-475

9. De Wispelaere A (2005) Ovoids and spreads of finite classical generalized hexagons and applications. Ph.D. thesis, Ghent University

10. El Zahar M, Sauer NW (1985) The chromatic number of the product of two 4-chromatic graphs is 4 . Combinatorica 5:121-126

11. Fiala NC, Haemers WH (2006) 5-chromatic strongly regular graphs. Discrete Math 306:3083-3096

12. Fon-Der-Flaass DG (1993) There exists no distance-regular graph with intersection array $(5,4,3 ; 1,1,2)$. Eur J Combin 14:409-412 
13. Garey MR, Johnson DS, Stockmeyer LJ (1976) Some NP-complete graph problems. Theor Comput Sci $1: 237-267$

14. Godsil CD (2004-2006) Interesting graphs and their colourings, unpublished notes 2004-2006. http://quoll.uwaterloo.ca/pstuff/colours.pdf

15. Haemers WH (1979) Eigenvalue techniques in design and graph theory. Ph.D. thesis, Eindhoven University of Technology (http://alexandria.tue.nl/extra3/proefschrift/PRF3A/7909413.pdf) Also: Math Centre Tract 121, Mathematical Centre, Amsterdam, 1980

16. Hoffman AJ (1970) On eigenvalues and colorings of graphs. In: Harris B (ed) Graph theory and its applications. Acad. Press, New York, pp 79-91

17. Ivanov AA, Shpectorov SV (1990) The $P$-geometry for $M_{23}$ has no nontrivial 2 -coverings. Eur. J Combin 11:373-379

18. Payan C (1992) On the chromatic number of cube-like graphs. Discrete Math 103:271-277

19. Sokolová M (1987) The chromatic number of extended odd graphs is four.. Časopis Pěst Mat 112: 308-311 